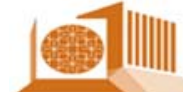

كلية الهندسة College of Engineering QATAR UNIVERSITY,

\section{Drones for Smart Agriculture}

Alaa Alnakeeb

Orob Balaawi

Noor Ellithy
Qatar University

Electrical Engineering Department

Advisor: Dr. Nizar Zorba

\title{
Abstract
}

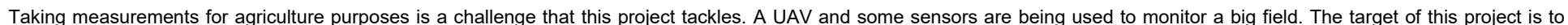

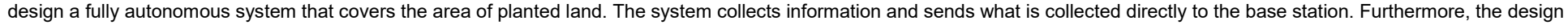

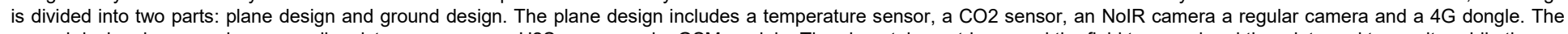

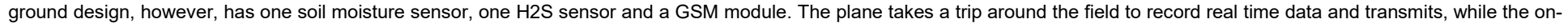
ground black box sends data to the base station. Finally, data is analysed, and reports are sent to the base station and the mobile application created for this cause.

\section{Introduction:}

This project will include a combination of sensing, scenario realization mechanisms, electronics implementations and communications to a central hub of the collected information. The students will design protocols for data collection by deciding on the proper sensors to load on the drone, as well as dealing with their constraints, especially in terms of weight (critical for flying) and power consumption (critical for battery drainage).

\section{The objectives of this project are: \\ * Fix and equip the plane \\ * Implement a fully autonomous system \\ * Monitor and gather real-time data}

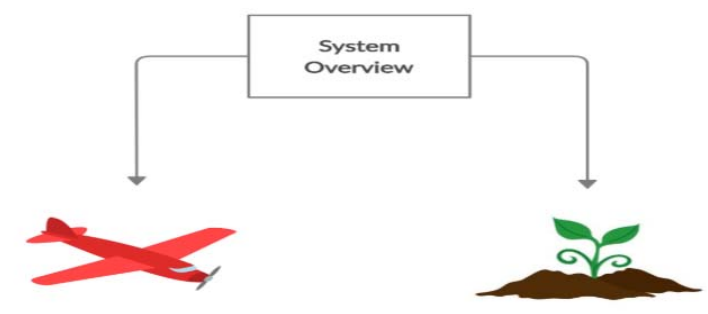

\section{On-Ground System}

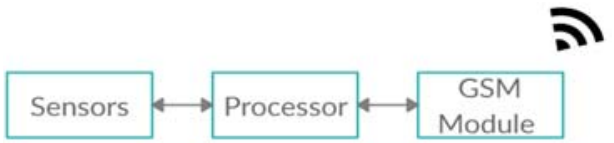

The On-ground design can be seen in the figure. The Black Box design consists of sensors and a GSM unit connected to an Arduino. Positioning this box on the ground was due to the need to be close to the soil and plants to get information that is as accurate as possible.

\section{Implementation}

\section{i. Mobile Application}

As part of the implementation of the project, a mobile application is created to allow the end user to remotely monitor the system. Data gathered from the sensors will be passed to the application which is created through the android studio platform. Moreover, the application will allow the user to monitor five different parameters. The Notification Centre notifies the user in case of an emergency or a sudden change in one of the parameters.

\section{ii. On-ground}

Several units of the black box will be arranged across the farm. This is because, the soil moisture and hydrogen Sulfide levels differ from one region to another. Therefore, the area that will be covered will be divided into zones where one box is installed in each zone Depending on the flight time, the plane will cover an area of about $28 \mathrm{~km}$. Therefore, the total area of the farm is $49 \mathrm{~km}^{2}$. Since the soil shows insignificant changes within $1 \mathrm{~km}$, the $49 \mathrm{~km}^{2}$ area will be divided into 49 zone where each zone of area $1 \mathrm{~km}^{2}$. Therefore, 49 boxes will be spread across the farm.

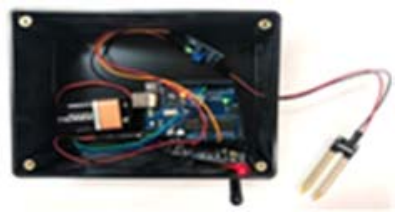

Drone

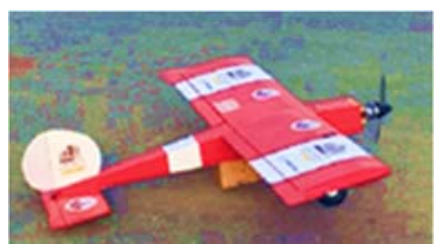

On-Plane System

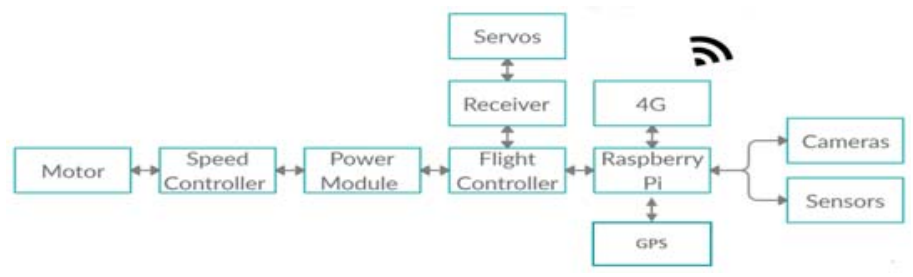

The on-plane design as seen in the figure consists of both mechanical and electrical systems. The mechanical system is responsible for flight control where the electrical system is for data collection and farm monitoring.

\section{Conclusions}

The proposed design consists of hardware and software sections. In the hardware, Sensors such as temperature, carbon dioxide, soil moisture and Hydrogen Sulphide are utilized to collect data for the user. The chosen sensors were picked based on a selection criterion that required low power consumption, applicable weight, and measurement accuracy. After selecting the appropriate sensors, a circuit design was implemented as seen in figures. On the other hand, the software design consists of a mobile phone application to alert the user with the data collected and to allow the user to control some aspects. By combining the hardware and software designs, the user can be completely involved in the agriculture process with the use of the UAV on its own.

\section{Future Work}

The circuits will be implemented as per the proposed design therefore, the full hardware system could be tested. Moreover, codes will be tested once they are uploaded into the microcontrollers and the full system is implemented. After all these tasks are done, the plane will be taken to be tested and data will be collected for analysis from an actual farm.

\section{References}

[1]Lyon, D. (2007). Surveillance studies: An overview.

[2]Polity. Lyon, D. (Ed.). (2006). Theorizing surveillance. Routledge.

[3]What are the techniques of surveillance? - Red Deer, Edmonton \& Calgary | Alberta \& British Columbia. (2019).

[4]How to Automate Surveillance Easily with Deep Learning. (2019).

[5]Andersen, R. (2019). An Eye Without an 'I': Justice and the Rise of Automated Surveillance. 